\title{
The Unusual Improvement of Intermittent Claudication Distance in Patient with Peripheral Arterial Disease Utilizing "2/3 Claudication Distance" Protocol of Exercise Training
}

\author{
Adam Staron, Mohammed Al-Subaie, Jana Al-Qahtani \\ Cardiac Rehabilitation Unit, Adult Cardiology Department, Prince Sultan Cardiac Center, Riyadh, KSA \\ Email: astaron1973@wp.pl
}

How to cite this paper: Staron, A., Al-Subaie, M. and Al-Qahtani, J. (2020) The Unusual Improvement of Intermittent Claudication Distance in Patient with Peripheral Arterial Disease Utilizing " $2 / 3$ Claudication Distance" Protocol of Exercise Training. Case Reports in Clinical Medicine, 9, 29-34.

https://doi.org/10.4236/crcm.2020.91005

Received: November 6, 2019

Accepted: January 5, 2020

Published: January 8, 2020

Copyright ( 2020 by author(s) and Scientific Research Publishing Inc. This work is licensed under the Creative Commons Attribution International License (CC BY 4.0).

http://creativecommons.org/licenses/by/4.0/ (c) (i) Open Access

\begin{abstract}
Peripheral arterial disease (PAD) affects $12 \%-20 \%$ of the population over 65 years. PAD is a component of systemic atherosclerosis and is associated with increased rate of all-cause mortality and cardiovascular events, compared with those without PAD. The strongest risk factors for PAD are older age and cigarette smoking. PAD accelerates functional decline leading to physical disability. Many randomized clinical trials demonstrated that treadmill walking training increases pain-free and maximal walking distances in patients with PAD. Exercise intervention is strongly recommended for patients with PAD and symptoms of intermittent claudication (IC). The need to provide an exercise training program to patients with peripheral arterial disease is essential, as almost half of these patients will eventually develop coronary artery disease. There are many existing treadmill walking protocols for patients with PAD. Most of these are based on claudication severity. We present a case of significant improvement of pain-free distance and maximum treadmill walking distance in a patient with PAD after 12 weeks of walking training program utilizing the " $2 / 3$ claudication distance" formula.
\end{abstract}

\section{Keywords}

Peripheral Arterial Disease, Intermittent Claudication, Aerobic Training, Cardiac Rehabilitation

\section{Introduction}

Cardiac rehabilitation (CR), according to the WHO guidelines is defined as "the 
comprehensive and coordinated use of medical, social, educational and professional resources to accommodate patients to a new lifestyle and enable them to achieve the best performance" [1]. The cardiac rehabilitation program consists of physical exercise sessions, dietary counselling, educational classes on lifestyle changes and psychosocial support to patients and their families. Supervised exercise training remains a core component of the cardiac rehabilitation program, focusing on aerobic, resistance, neuromotor and flexibility components. Cardiac rehabilitation is recommended for patients with PAD and claudication, as it reduces symptoms, prolongs claudication distance and improves quality of life [2]. The goals of a comprehensive cardiac rehabilitation program in cases of patients with PAD include symptoms reduction, improvement of exercise capacity and a decrease in the risk of cardiovascular events. Cardiac rehabilitation program outcome measures include exercise capacity, modifiable risk factors (blood lipid and glucose levels, blood pressure, smoking), psychological outcomes (depression, anxiety), return to work and quality of life. All of them can be easily quantified and interpreted. Functional capacity assessment includes maximum work-load from exercise test (as peak oxygen uptake in $\mathrm{ml} / \mathrm{kg} / \mathrm{min}$ or as a value in metabolic equivalents) or change in distance achieved on a 6-minute walking. A meta-analysis of 21 studies performed in 1995 concluded that a supervised exercise improved maximum walking distance on a treadmill by $122 \%$ and pain-free walking distance on a treadmill by $179 \%$ [3]. Benefits of exercise training exist also among patients with PAD who do not have claudication. The disputable question remains as to which walking treadmill protocols are optimal and most effective for patients with PAD.

\section{Case Report}

A 59-year-old male patient, active smoker (half a pack a day for 20 years), with peripheral arterial disease and 100 meters claudication distance has been admitted to the Cardiac Rehabilitation Unit at Prince Sultan Cardiac Center, Riyadh. The Patient had a history of coronary artery disease (anterior wall myocardial infarction and percutaneous coronary angioplasty with stent implantation in 2016, coronary artery bypass graft surgery in 2018), hypertension and dyslipidemia. The patient's medication regimen included aspirin $75 \mathrm{mg}$ once a day, diltiazem $120 \mathrm{mg}$ twice a day, losartan $50 \mathrm{mg}$ once a day and atorvastatin, $40 \mathrm{mg}$ once a day Upon admission the patient complained of pain in his calves during walking. No recent history of chest pain or shortness of breath during daily activities. At physical examination his heart rate, blood pressure and oxygen saturation were within normal limits. Lung examination without alterations. Heart assessment revealed a grade 2 systolic murmur over the apex area. Resting ankle-brachial index (ABI) was 0.60 and 0.70 at the right and left dorsalis pedis, respectively. Transthoracic echocardiography revealed moderately impaired left ventricular systolic function with ejection fraction (EF) of $45 \%$. Graded stress test on treadmill utilizing modified Bruce protocol had been stopped due to leg 
fatigue after reaching 5.0 MET, which is indicative of a low functional capacity according to the American Association of Cardiovascular and Pulmonary Rehabilitation guidelines. At a separate session the treadmill walking protocol with 3 $\mathrm{km} / \mathrm{h}$ speed and no inclination had been performed with calculated distances to onset of claudication and walking was ceased due to unbearable pain at 100 and 150 meters subsequently. For exercise training purposes we utilized the " $2 / 3$ claudication distance" walking treadmill protocol. The patient covers $2 / 3$ of the calculated maximal treadmill walking distance. One cycle consists of three such walks, i.e. $3 \times 2 / 3$ maximal walking distance, with a 2 -minute rest between each. Full training consists of three such cycles with 10 minutes break between each. Ideally the patient should perform such training three times a day, most days of the week. Such a program should be continued for at least 3 - 4 months. In cases where pain occurs, rest periods should be taken until pain subsides and subsequent walking blocks should be adjusted accordingly. In this case the patient repeated such training two times more during exercise days and three times during non-exercise days in addition to his supervised sessions. After 12 weeks of training, the distances attained prior to the onset of pain and to unbearable pain significantly increased to 420 and 585 meters respectively. Controlled ABI increased by 0.1 at the right and left dorsalis pedis respectively and controlled graded stress test had been terminated due to leg pain after reaching 6.5 MET.

\section{Discussion}

Exercise intervention is strongly recommended for patients who present with peripheral artery disease. Regular exercise sessions reduce symptoms, prolong claudication distance and improve quality of life in patients with PAD. It must be remembered that patients with PAD and claudication have about $50 \%$ reduction in peak oxygen consumption in comparison with age-matched individuals [4]. It affects their functional status, but also their quality of life and they often start to avoid physical activity, especially walking, which in turn leads to further decline in functional status. Patients with PAD present also with higher prevalence of depression [5]. As mentioned earlier, meta-analysis from 1995 revealed increase of maximal walking distance by $122 \%$ and pain-free walking distance by $180 \%$ [6]. Systematic review of controlled clinical trials (22 studies with over 1200 participants conducted by the Cochrane group in 2008 compared programs based on supervised exercise training with usual care), concluded that exercise significantly increased walking time (by 5 minutes) and walking distance (by $100 \mathrm{~m}$ ). Degree of exercise-related improvement varies across studies, depending on intensity of exercise training as well as adherence to exercise programs. Nevertheless, all existing studies have shown exercise-related improvements. Mechanisms of such improvement have been explained by improved skeletal muscles metabolism, rheological changes and blood redistribution [7]. It includes reduction of post-exercise lactates production, reduced blood viscosity, and increased production of endothelial nitric oxide. Potential 
neovascularization or dilation of existing collaterals has been also postulated. Exercise training in cases of patients with PAD should have intensity set between $40 \%$ and $70 \%$ of heart rate reserve and should last for at least $20-30$ minutes (without specified accurate time of a day). Weight-bearing exercises such walking are preferred. There are many existing walking treadmill protocols dedicated to the patients with PAD, e.g. Larsen protocol with a constant speed of $4.6 \mathrm{~km} / \mathrm{h}$ and grade adjustment or protocol by Dahloff with speed adjustment (starting from $4 \mathrm{~km} / \mathrm{h}$ ) and no grade. Some centers utilize protocols with both speed and inclination change [8]. In general, trainings are conducted to onset of pain or when pain becomes moderate-to-severe. In studies comparing both types of training significant improvement in walking distance within both groups has been demonstrated, with no statistical differences between them. Typically exercise programs for patients with PAD consist of 3 supervised sessions per week with a minimum of 20 - 30 minutes of exercise. Patients usually are asked to walk to the moment when pain is maximal. Such programs usually last $4-6$ months. There is a general agreement that training progression should be gradual to enable adaptation to training stimulus. This can be achieved by change of training components, i.e. the duration of walking or rest periods, workload (treadmill speed and/or grade), or frequency. In most cases, total exercise volume is increased by alternative increase in duration and workload [9]. Optimal walking training duration is disputable. Meta-analysis by Fakhry demonstrated beneficial effects of walking training regardless of program duration, i.e. 4 - 11 weeks, $12-26$ weeks and $>26$ weeks. Improvement in treadmill walking performance occurs within $4-8$ weeks and benefits are achieved usually by $10-12$ weeks [10]. In a trial trainings ranged in duration from 4 to 104 weeks (more than half had durations between 12 and 26 weeks). Whereas we strictly followed the model of 3 supervised sessions per week, lasting at least 30 minutes each, with a total program duration of 12 weeks, both training protocols and training progression at our center vary. We consistently commence with precise calculation of distances to onset of claudication and to unbearable pain. Based on these we start the exercise walking treadmill sessions utilizing one of existing protocols (eg. Dahloff, Larsen or " $2 / 3$ claudication distance" formula). In a case of " $2 / 3$ claudication distance" training protocol, we commence the first treadmill walking sessions with constant speed (usually it is $3.0 \mathrm{~km} / \mathrm{h}$ or $3.5 \mathrm{~km} / \mathrm{h}$ for a taller individuals) and no inclination. Such a speed is similar to many existing walking protocols. i.e. $3.2-4.0 \mathrm{~km} / \mathrm{h}$ ). Walking time is calculated to cover approximately $2 / 3$ of maximal walking distance from the test. In this case, our patient training program consisted of 5 such walking blocks with 2 min rests (or until pain subsides) three times a week. Once a week after the fifth block and subsequent 10 min rest, we performed the test with new calculation of distances to onset of pain and to unbearable pain. Then the duration of blocks for the next sessions had been recalculated based on test results. As mentioned earlier, improvement of maximal walking distance as well as distance to onset of pain in case of this 
patient reached over $300 \%$, which is significant in comparison with data from the literature. What we found as a real advantage of $2 / 3$ claudication formula is the absence of severe pain. Covering $2 / 3$ claudication distance rarely provokes maximal pain. If present, usually a mild degree and well tolerated pain occurred which did not affect the overall training schedule. One of potential pitfalls of protocols till moderate to severe pain is low program adherence, related to pain intolerance. i.e. some patients reduce the number of training sessions or even avoid walking. Regular (i.e. once a week) recalculation of distance to onset of claudication and to maximal walking distance allows for adequate training progression by increasing duration of walking blocks, without workload change. In practice we used constant speed ( 3.0 or $3.5 \mathrm{~km} / \mathrm{h}$ ) with no grade, and increased training duration. From our experience " $2 / 3$ claudication distance" formula seems to be a feasible and very effective exercise intervention for patients with peripheral arterial disease and intermittent claudication.

\section{Conflicts of Interest}

The authors declare no conflicts of interest regarding the publication of this paper.

\section{References}

[1] American Association of Cardiovascular and Pulmonary Rehabilitation (2013) Guidelines for Cardiac Rehabilitation and Secondary Prevention Programs. 5th Edition, Human Kinetics Publishers, Champaign, IL.

[2] Gardner, A.W., Katzel, L.I., Sorkin, J.D. and Goldberg, A.P. (2002) Effects of Long-Term Exercise Rehabilitation on Claudication Distances in Patients with Peripheral Arterial Disease: A Randomized Controlled Trial. Journal of Cardiopulmonary Rehabilitation, 22, 192-198. https://doi.org/10.1097/00008483-200205000-00011

[3] Gardner, A.W. and Poehlman, E.T. (1995) Exercise Rehabilitation Programs for the Treatment of Claudication Pain: A Meta-Analysis. JAMA, 274, 975-980. https://doi.org/10.1001/jama.1995.03530120067043

[4] Bauer, T.A., Brass, E.P., Nehler, M., Barstow, T.J. and Hiatt, W.R. (2004) Pulmonary $\mathrm{VO}_{2}$ Dynamics during Treadmill and Arm Exercise in Peripheral Arterial Disease. Journal of Applied Physiology, 97, 627-634. https://doi.org/10.1152/japplphysiol.00612.2003

[5] Hamburg, N.M. and Balady, G.J. (2011) Exercise Rehabilitation in Peripheral Arterial Disease: Functional Impact and Mechanisms of Benefits. Circulation, 123, 87-97. https://doi.org/10.1161/CIRCULATIONAHA.109.881888

[6] Fakhry, F., van de Luijtgaarden, K.M. and Bax, L. (2012) Supervised Walking Therapy in Patients with Intermittent Claudication. Journal of Vascular Surgery, 56, 1132-1142. https://doi.org/10.1016/j.jvs.2012.04.046

[7] Tan, K.H., Cotterell, D. and Sykes, K. (2000) Exercise Training for Claudicants: Changes in Blood Flow, Cardiorespiratory Status, Metabolic Functions, Blood Rheology and Lipid Profile. Eur J Vasc Endovasc Surg, 20, 72-78. https://doi.org/10.1053/ejvs.2000.1137

[8] Bromboszcz, J. and Dylewicz, P. (2015) Rehabilitacja Kardiologiczna-Stosowanie 
ćwiczeń fizycznych. Elipsa-Jaima.

[9] Treat-Jacobson, D., McDermott, M., Bronas, U., Campia, U., Collins, T. and Criqui, M. (2019) Optimal Exercise Programs for Patients with Peripheral Artery Disease: A Scientific Statement from the American Heart Association. Circulation, 139, 10-33. https://doi.org/10.1161/CIR.0000000000000623

[10] Gardner, A.W., Montgomery, P.S. and Parker, D.E. (2012) Optimal Exercise Program Length for Patients with Claudication. Journal of Vascular Surgery, 55, 1346-1354. https://doi.org/10.1016/j.jvs.2011.11.123 